\title{
ON SCHACHERMAYER'S EXAMPLE ABOUT THE BANACH-SAKS PROPERTY
}

\author{
by CARMELO NUÑEZ†
}

(Received 9 January, 1989)

1. Introduction. A Banach space $(X,\|\cdot\|)$ is said to have the Banach-Saks property (B.S.P.) if, for every bounded sequence $\left(x_{n}\right)$ in $X$, we can choose a subsequence $\left(x_{n}^{\prime}\right)$ of $\left(x_{n}\right)$ such that the sequence

$$
\left(y_{n}\right)=\left(\left(x_{1}^{\prime}+\ldots+x_{n}^{\prime}\right) / n\right)
$$

converges in the $X$-norm. This property, that a Banach space may enjoy or not, has been extensively studied.

On the other hand, we recall that $L^{2}([0,1], X)$, which we shall refer to as $L^{2}(X)$, is the Banach space of the Bochner measurable functions from $[0,1]$ to $X$, with the norm

$$
\|f\|_{2}=\left\{\int\|f(t)\|^{2} d t\right\}^{1 / 2} .
$$

We use [3] as our reference for $L^{2}(X)$ spaces.

It is known that $L^{2}([0,1])$ has the B.S.P. Nevertheless there are examples (the first ones are due to J. Bourgain and W. Schachermayer) of Banach spaces $X$ which have the B.S.P. but such that $L^{2}(X)$ does not. The example of Professor W. Schachermayer seems to be the easiest, and has been neatly described in $[1$, p. 152]. Our aim is to present a slight refinement of known results about this space that we will call $\left(B_{1},\|\cdot\|\right)$, as in [1] . There it is shown that there exists a sequence $\left(f_{n}\right) \subset L^{2}(X)$ which satisfies:

(a) $\left\|f_{n}(t)\right\|=1,\left(f_{n}(t)\right) \stackrel{\leftrightarrow}{\rightarrow} 0$ for every $t \in[0,1]$ and therefore $\left(f_{n}\right) \stackrel{M}{\rightarrow} 0$;

(b) for each $t \in[0,1]$, there exists an increasing sequence of integers $(n(k))$-this sequence depending on $t$-such that for every subsequence $\left(f_{n(k)}^{\prime}\right)$ of $\left(f_{n(k)}\right)$,

$$
\frac{1}{m}\left\|\sum_{k \leq m} f_{n(k)}^{\prime}(t)\right\| \rightarrow 0 \quad \text { as } m \rightarrow \infty
$$

(c)

$$
\lim _{u \rightarrow \infty} \inf \left\{\frac{1}{k}\left\|\sum_{i \leq k} \varepsilon_{i} f_{n(i)}\right\|_{2}: u \leq n(1)<\ldots<n(k), \varepsilon_{i}= \pm 1\right\}=1,
$$

for every $k \in \mathbb{N}$.

(We note that (b) follows from (a) and the fact that $B_{1}$ has the B.S.P.) Our improvement is as follows.

(d) For every increasing sequence of integers $(n(k))$

$$
\mu\left(\left\{t \in[0,1]: \lim _{k} \frac{1}{k}\left\|\sum_{i \leq k} \varepsilon_{i} f_{n(i)}(t)\right\|=1\right\}\right)=1,
$$

where $\varepsilon_{i}= \pm 1$ and $\mu$ is Lebesgue measure.

† Supported in part by CAICYT grant 0338-84. The author wishes to thank Professors F. Bombal, J. Diestel and the referee for their advice.

Glasgow Math. J. 32 (1990) 201-203. 
The result (d) should be compared to previous ones of, for example, [2], [4] and [7]. There, if $\left(g_{n}\right):[0,1] \rightarrow Y$, and for every $t \in[0,1]$ we can choose a sequence $(n(k))$ depending on $t$-such that $\left(g_{n(k)}(t)\right)$ satisfies "something" then we can find a sequence of integers $(m(k))$ such that $\left(g_{m(k)}\right)$ satisfies "something" a.e.

2. Proof of (d). We use the terminology of $[1$, p. 152]. In order to simplify the notation we shall say that the set $\left\{e_{n}: n \in A\right\}$ is totally admissible if $A \subset \mathbb{N}$ is totally admissible.

First, we show that

$$
\mu\left(\left\{t \in[0,1]: \lim _{k} \frac{1}{k}\left\|\sum_{i \leq k} \varepsilon_{i} f_{i}(t)\right\|=1\right\}\right)=1 .
$$

Let $r: \mathbb{N} \rightarrow \mathbb{N}$ be any function satisfying:

(a) $r(k) / k \rightarrow 0$ as $k \rightarrow \infty$,

(b) $\sum_{k} k(k+1) / 2^{r(k)}<\infty$.

(Here $r(n)=[\bigvee n]$, where [.] denotes the integer part of any real number, will do.) We want the following equality. If $B_{k}=\left\{t \in[0,1]:\left\{f_{r(k)}(t), \ldots, f_{r(k)+j}(t), \ldots, f_{k}(t)\right\}\right.$ is a totally admissible set $\}$, then

$$
b(k)=\mu\left(B_{k}\right)=\prod_{j=0}^{k-r(k)}\left(1-j / 2^{r(k)}\right) .
$$

To prove (**), we define (for any $j=0, \ldots, k-r(k)) g_{j}(t)$ to be the unique element of $\mathbb{N}$ such that

(a) $2^{r(k)} \leq g_{j}(t)<2^{r(k)+1}$,

(b) $t(r(k)+j) \in\left[g_{j}(t) / 2^{r(k)},\left(g_{j}(t)+1\right) / 2^{r(k)}[\right.$.

Then it is clear that the condition $t \in B_{k}$ is equivalent to $g_{i}(t) \neq g_{j}(t)$ if $i \neq j$. Due to the fact that $\left\{g_{j}: j=0, \ldots, r(k)\right\}$ is a set of independent random variables, we obtain $(* *)$.

Note now that

$$
\left\{t \in[0,1]: \lim _{k}\left\|\sum_{i \leq k} \varepsilon_{i} f_{i}(t)\right\|=1\right\} \supset \bigcup_{n}\left(\bigcap_{j>n} B_{j}\right) .
$$

In fact, if $t \in \bigcap_{j>n} B_{j}$, then for $k>n$, we have

$$
\frac{1}{k}\left\|\sum_{i \leq k} \varepsilon_{i} f_{i}(t)\right\| \geq \frac{1}{k}\left\|P_{k}\left(\sum_{i \leq k} \varepsilon_{i} f_{i}(t)\right)\right\|,
$$

where $P_{k}$ is the projection on the totally admissible set

$$
A_{k}(t)=\left\{n(j): f_{r(k)+j}(t)=e_{n(j)}, j=0, \ldots, k-r(k)\right\},
$$

and so

$$
\frac{1}{k}\left\|\sum_{i \leq k} \varepsilon_{i} f_{i}(t)\right\| \geq \frac{1}{k}\left(\sum_{r(k) \leq i \leq k}\left\|\varepsilon_{i} f_{i}(t)\right\|\right)=(k-r(k)+1) / k .
$$


Observing that $\log (1-x) \geq-2 x$ if $0 \leq x<\frac{1}{2}$, we have, for $k$ sufficiently large,

$$
\log (b(k)) \geq-2 \sum_{j \leq k-r(k)} j / 2^{r(k)} \geq-k(k+1) / 2^{r(k)}
$$

Now, using the fact that $1-e^{-x} \leq x$ if $x>0$, it is clear that $\sum_{k}(1-b(k))<\infty$. We deduce that $\mu\left(\bigcup_{n}\left(\bigcap_{j>n} B_{j}\right)\right)=1$, and so (*) is proved.

Finally, we prove (d). For every increasing sequence of integers $(n(k))$, we let $B_{k}=\left\{t \in[0,1]:\left\{f_{n(r(k))}(t), \ldots, f_{n(r(k)+j)}(t), \ldots, f_{n(k)}(t)\right\}\right.$ is a totally admissible set $\}$.

Obviously the set $B_{k}$ depends on the sequence of integers $(n(k))$. Then we obtain

$$
\mu\left(B_{k}\right)=\prod_{j=0}^{k-r(k)}\left(1-j / 2^{n(r(k))}\right) \geq \prod_{j=0}^{k-r(k)}\left(1-j / 2^{r(k)}\right) .
$$

The last inequality holds since $n(i) \geq i$. It only remains for the reader to repeat the analysis of the case $n(k)=k$.

Remark. The reader should note that the generalization of a property related to the Césaro summation method to other summation methods is straightforward if the convergence that we are studying is the norm convergence of a Banach space (see [5] and $[1$, p. 58]), but the convergence a.e. is not of this kind. Nevertheless we can obtain (with the notation of [5]) the following result.

$\left(\mathrm{d}^{\prime}\right)$ For every $A$ u.a.n.r.s.m., and for every sequence of integers $(n(k))$, we have

$$
\mu\left(\left\{t \in|0,1|: \lim _{k} \inf \left\|\sum_{i<\infty} a_{k i} \varepsilon_{i} f_{n(i)}(t)\right\| \geq 1\right\}\right)=1 .
$$

The proof is similar and we omit it.

\section{REFERENCES}

1. B. Beauzamy and J. T. Lapreste, Modèles étalés des espaces de Banach, (Hermann, Paris, 1984).

2. J. Bourgain, An averaging result for $C_{0}$-sequences, Bull. Soc. Math. Belg. 30 (1978), 83-87. 1977)

3. J. Diestel and J. J. Uhl, Jr. Vector measures, Surveys of the Amer. Math. Soc. 15, (A.M.S.,

4. G. Pisier, Une propriété de stabilité de la classe de spaces ne contenant pas $l^{1}$, C.R. Acad. Sci. Paris. Sér, A 286 (1978), 747-749.

5. H. P. Rosenthal, Weakly independent sequences and the Banach-Saks property, Bull. London Math. Soc. 8 (1976), 22-24.

6. W. Schachermayer, A Banach space $E$ such that $L^{2}(E)$ is not Banach-Saks, Israel J. Math. 40 (1981), 340-344.

7. M. Talagrand, Weak Cauchy sequences in $L^{\prime}(E)$, Amer. J. Math. 106 (1984), 703-725.

Departamento de Analisis Matematico

Facultad de Matematicas

UNIVERSIDAD COMPLUTENSE

28040-MADRID, SPAIN 